\title{
PERFIL DE LAS LICENCIATURAS EN ARTE DEL SISTEMA UNIVERSITARIO CHILENO
}

\section{Ignacio Villegas ${ }^{1}$}

\section{RESUMEN}

Este trabajo expone los principales rasgos que caracterizan hoy a los trece programas de licenciatura en arte del sistema universitario chileno, mediante el análisis de la información entregada por informantes directos, Servicio de Información de Educación Superior, SIES, y sitios web institucionales. A partir de ello, es posible diseñar un perfil de los programas, cuyos rasgos principales son la poca diferenciación de estructura curricular existente entre ellos; las grandes diferencias en el valor de las matrículas; el alto número de estudiantes mujeres; el reducido número de académicos doctores, y las acciones de reforzamiento para facilitar el proceso de graduación. El análisis de los datos constituye un insumo para el diseño de políticas en la enseñanza del arte en las universidades chilenas.

Palabras clave: arte, bellas artes, escuela de arte, programas pregrado

\section{PROFILE OF ARTS BACHELOR'S DEGREE IN THE CHILEAN UNIVERSITY SYSTEM}

\section{ABSTRACT}

This work shows the main features that shape the 13 undergraduate art programs in the new Chilean university system by analyzing information provided by direct informants, SIES and official Web pages. From this, it is possible to display a profile for these programs, whose main features are a little differentiation in terms of curricular structure, differences in tuition fees rates, a high number of women as students, a reduced number of faculty members with a $P h D$., and actions towards facilitating the graduation process. Analysis of data constitutes a basis for designing policies for teaching arts in Chilean universities.

Keywords: art, fine arts, school of art, undergraduate programs

1 Profesor titular de la Escuela de Arte, Facultad de Artes, Universidad Católica de Chile. Santiago. Chile. Contacto: ivillega@uc.cl. 


\section{PERFIL DE LAS LICENCIATURAS EN ARTE DEL SISTEMA UNIVERSITARIO CHILENO}

\section{Introducción}

El presente artículo expone de forma comparativa el estado de los programas de licenciatura en arte del sistema universitario chileno, permitiendo el diseño de un perfil de estos. Metodológicamente, debemos señalar que la información incluida en el estudio coteja datos de las universidades que ofrecen la carrera de licenciatura en artes - u otra denominación equivalente- provenientes del Consejo de Rectores de las Universidades Chilenas (CRUCH) y del Servicio de Información de Educación Superior (SIES), más la información institucional alojada en las páginas web de los distintos programas. En las fuentes consultadas, los datos más completos y precisos son los del año 2008, no obstante se ha cotejado la información con datos más recientes alojados en las fuentes mencionadas. Cabe señalar que en la web los programas no contienen información completa, o bien los datos no están actualizados, debiendo recurrirse, en esos casos, a informantes directos (profesores o autoridades académicas). De esa forma, observamos que en la red las listas de académicos muestran una desactualización mayor en diciembre del año 2011. Así mismo, ha resultado complejo establecer equivalencias en esas planillas, ya sea por el carácter de las contrataciones (de planta o a honorarios), que no siempre se explicitan, o bien por las diferentes denominaciones de categorías académicas en cada institución.

Debemos indicar que se han considerado como equivalentes los programas bajo la categoría OCDE Arte y Arquitectura, que se denominan: licenciatura en Arte; licenciatura en Artes Visuales; licenciatura en Arte mención Artes Plásticas; licenciatura en Artes y Fotografía y licenciatura en Arte y Cultura Visual. Estos títulos se refieren a programas universitarios para la formación de graduados con dominios técnicos-instrumentales; conocimientos de historia; metodología de investigación y epistemología de cultura visual contemporánea. 
Con relación a la Universidad Metropolitana de Ciencias de la Educación (UMCE), hay un aspecto que puede llamar a confusión. Los estadígrafos muestran la carrera de licenciatura en Arte y la carrera de licenciatura en Educación Artística y pedagogía en Artes Visuales; la primera consiste en un programa especial creado en 1996 y corresponde al área OCDE Arte y Arquitectura, mientras la segunda corresponde al área Educación, por lo que esta última ha sido excluida de la lectura de los datos. No obstante la presentación que hace el Departamento de Artes Visuales de esta universidad en su sitio institucional señala:

"Esta unidad académica, destaca el interés por constituirse en una opción de calidad en la formación artística visual y artística educativa visual, instalándose como un espacio de formación para los profesores de Artes Visuales que requiere el sistema nacional de educación, así como ser una alternativa importante a la demanda de formación artística visual -'licenciados en artes visuales'- personas que optan por hacer su vida profesional en el campo de la creación en las Artes Visuales, con énfasis en la producción de obra personal y/o la gestión cultural visual, entendida epistemológicamente en el ámbito de la cultura visual y el pensamiento contemporáneo"2.

Los programas de licenciatura en arte, o equivalentes, incorporados a este estudio son impartidos por las siguientes instituciones (en orden alfabético):

1. Pontificia Universidad Católica de Chile (PUC)

2. Pontificia Universidad Católica de Valparaíso (PUCV)

3. Universidad Austral de Chile (UACH)

4. Universidad Católica de Temuco (UCT)

5. Universidad de Arte y Ciencias Sociales (ARCIS)

6. Universidad de Artes, Ciencias y Comunicación (UNIACC)

7. Universidad de Chile (UCH)

8. Universidad de Concepción (UDEC)

2 Universidad Metropolitana de Ciencias de la Educación. Facultad de Artes y Educación Física. Presentación. Disponible en http://www.umce.cl/ aplasticas/ 
9. Universidad de Playa Ancha de Ciencias de la Educación (UPLA) 10. Universidad del Desarrollo (UDD)

11. Universidad Diego Portales (UDP)

12. Universidad Finis Terrae (UFT)

13. Universidad Mayor (UMA)

14. Universidad Metropolitana de Ciencias de la Educación (UMCE) 15. Universidad Nacional Andrés Bello (UNAB)

Tabla n. ${ }^{\circ}$ 1. Datos generales de los programas de licenciatura en arte.

\begin{tabular}{|l|l|c|c|c|c|}
\hline Universidad & Nombre carrera & $\begin{array}{c}\text { Año } \\
\text { creación }\end{array}$ & $\begin{array}{c}\text { Vacantes por } \\
\text { semestre }\end{array}$ & $\begin{array}{c}\text { Duración } \\
\text { años }\end{array}$ & Comuna \\
\hline PUC & Lic. en Arte & 1959 & 75 & 4 & Providencia \\
\hline PUCV & Lic. en Arte & 1968 & 30 & 4 & Valparaíso \\
\hline UACH & Lic. en Artes Visuales & 2005 & 30 & 4 & I. Teja. Valdivia \\
\hline UCT & Lic. en Artes Visuales & 1980 & 30 & 4 & Temuco \\
\hline ARCIS & Lic. en Arte y Cultura Visual & 1991 & 50 & 4,5 & Providencia \\
\hline UNIACC & Lic. en Artes Visuales y Fotografía & 1996 & $50 / 15$ & 5 & Ñuñoa \\
\hline UCH & Lic. Artes mención Artes Plásticas & 1879 & 78 & 5 & Ñuñoa \\
\hline UDEC & Lic. en Artes Visuales & 1972 & 60 & 4 & Concepción \\
\hline UPLA & Lic. en Arte & 1989 & 20 & 4 & Valparaíso \\
\hline UD & Lic. en Arte & 2009 & 20 & 4 & Las Condes \\
\hline UDP & Lic. en Artes Visuales & 2006 & 35 & 5 & Santiago \\
\hline UFT & Lic. en Artes Visuales & 1993 & 70 & 4 & Providencia \\
\hline UMA & Lic. en Artes Visuales & 2005 & 20 & 4,5 & Santiago \\
\hline UMCE & Lic. en Artes Visuales & 1996 & 20 & 4 & Ñuñoa \\
\hline UNAB & Lic. en Artes Visuales & 2003 & 30 & 4 & Las Condes \\
\hline
\end{tabular}

Fuente: Bases de datos SIES, Mineduc.

\section{Origen}

El Decreto con Fuerza de Ley $N^{\circ} 1$ de diciembre de $1980^{3}$, cambió definitivamente la perspectiva de la educación superior en Chile, pues posibilitó la creación de universidades privadas, hecho que multiplicó la oferta universitaria en casi todas las áreas del conocimiento.

Las artes visuales o artes plásticas no se mantuvieron ajenas al fenómeno, no obstante la creación de escuelas de arte de nivel

3 Ver http://www.leychile.cl/Navegar?idNorma=3394\&r=1. También, en Diario Oficial $\mathrm{N}^{\circ}$ 30.855. Santiago de Chile, enero 3 de 1981. 
universitario no ha sido veloz ni abundante: en 30 años solo se han creado 11 programas nuevos.

\section{Desarrollo de los programas}

Al grupo de escuelas o departamentos de arte de la UCH, PUC, PUCV y UDEC, preexistentes a la fecha de promulgación del DFL mencionado, se sumó la escuela de arte de la entonces sede Temuco de la PUC (1980); UMCE (1985); UPLA (1989); ARCIS (1991); UFT (1993) y UNIACC (1996). Cinco escuelas más se crean en la década del 2000: en la UNAB (2003); UMA y UACH (2005); UDP (2006) y UDD (2009).

En más o en menos, la estructura de los dos programas de más antigüedad (universidades PUC y UCH) fueron el modelo de trabajo académico de todos los programas creados en 30 años, no obstante y como parece lógico, todas han reestructurado sus mallas curriculares con el correr de los años, avanzando claramente en dos direcciones; por una parte -las menos-con programas que conservan la estructura de enseñanza que privilegia en sus mallas la formación disciplinaria de tipo técnico/instrumental, y por otra parte -las más- aquellas unidades académicas con programas que ubican como centro de sus procesos de enseñanza-aprendizaje la construcción de una obra sin importar la disciplina, la técnica o los instrumentos utilizados para materializarla, como se verá a continuación.

\section{Modelos curriculares}

En efecto, el Departamento de Arte de la UCH y la Escuela de Arte de la PUC, la primera con 133 años de experiencia ${ }^{4}$ y la segunda con 52, fueron los dos grandes referentes para la estructuración de los procesos de enseñanza-aprendizaje universitario en artes visuales en el nuevo sistema iniciado en 1980. Ambas compartían cinco rasgos similares: la duración de cinco años (10 semestres), la

4 El Estatuto Orgánico de 1879 crea la Facultad de Filosofía, Humanidades y Bellas Artes, de la cual dependerá la Academia de Bellas Artes, que luego pasa a denominarse Escuela de Bellas Artes. Consultado en: http://www.artes.uchile.cl/portal/artes-visuales/departamentode-artes-visuales/30345/resena-historica 
admisión anual, la estructura de semestres, el régimen diurno y el impartir las tradicionales cuatro disciplinas instrumentales: pintura, dibujo, escultura y grabado; esta estructura básica fue replicada en las nuevas escuelas. En otras palabras, no hay escuelas de arte de corte netamente técnico.

En 1994, la Escuela de Arte de la PUC cambia la orientación de su curriculum, eliminando las menciones, reduciendo la importancia de la técnica en la construcción de la obra y centrándose en el análisis epistemológico de los proyectos de obra de sus estudiantes, con clara influencia de la Universidad de París 1, que asesoró en este cambio realizado entre 1992 y 1995.

Este nuevo modelo aportó a las escuelas del sistema universitario nacional la idea de incorporar un curso central de discusión de los proyectos de obra, que acepta la construcción con técnicas mixtas y permite la exploración de nuevas formas de trabajo constructivo, más allá de las disciplinas tradicionales. El esquema de trabajo inaugurado en 1994, en que surge la noción de interdisciplina en la estructura del proceso de enseñanza-aprendizaje, genera un paso más complejo que la tradicional multidisciplina de la estructura administrativa de las unidades de arte instaurada desde la academia del siglo XIX. Por lo menos ocho escuelas mantienen esta fórmula hasta hoy, con talleres centrados en los procesos creativos y resultados de las obras de sus estudiantes. La nomenclatura empleada acusa la fórmula interdisciplinaria con la que se estructura la malla: taller de producción de obra (PUC); taller de plástica (PUCV); taller de producción de campo/taller de producción de borde (ARCIS); taller de proyectos (UNIACC); taller de lenguaje visual/taller central de arte (UCH); taller de producción visual (UDEC); taller central (UDP); taller de proyectos visuales/taller integrado (UMA). La incorporación de este rasgo en las mallas curriculares redujo el número de créditos de los talleres de disciplinas tradicionales, restando importancia a las técnicas de grabado, dibujo, pintura, escultura y fotografía, un logro para no exceder el límite habitual de 50 créditos por semestre.

La agregación de las tecnologías digitales a las mallas curriculares de los programas universitarios de arte comenzó hacia 
1995, con los primeros cursos de gráfica digital, situación que hoy es común para todos los curricula del sistema.

Por último, cabe mencionar que once programas han incorporado cursos, actividades, talleres o seminarios finales en la carrera para reforzar el término de los estudios y asegurar la graduación. Se trata de un espacio que permite al estudiante preparar el proyecto para su examen de grado, que consiste en una obra más un texto denominado memoria, para subrayar el análisis de la propuesta visual. Es un documento que contiene una exploración teórica sobre las ideas y dificultades de la obra, análisis de forma, imagen, montajes y técnicas. Esta fórmula facilita el aumento de la tasa de graduación de sus estudiantes. Tal es el caso de la PUC con Taller y memoria de grado 1 y 2; la UACH con Avance de tesis I y II; ARCIS con Seminario taller terminal; UCH con el Taller y seminario de preparación de trabajo de creación artística y Seminario de preparación de informe de trabajo de creación artística; la UDEC con Taller de grado 1 y 2; UPLA con Tesis; UDD con Taller de proyectos e investigación aplicada; la UDP con Seminario de título y pasantía; la UFT con Taller de proyectos y Tutoría 1 y 2; UMCE con Taller de creación y la UNAB con Seminario de titulación y memoria de grado.

\section{Académicos}

La mayoría del cuerpo académico que se desempeñó como docente de los programas de licenciatura en arte durante los primeros 15 años, desde la puesta en vigencia del DFL No $1 / 1980$, provenía mayoritaria y naturalmente de licenciados de la PUC y de la UCH. Este hecho bien pudo constituir un factor coadyuvante en la réplica de esos modelos de enseñanza. El cuerpo docente de la UCT, de la UDEC y de la PUCV se nutrió por algunos años de muchos académicos con título de profesor de Estado en Artes Plásticas o artífice. El giro hacia un plantel constituido por un número importante de posgraduados es observable solo en los últimos 20 años. Sin embargo, las cifras que exponen los respectivos programas muestran aún hoy una fuerte carencia en la contratación de doctores, lo que hace suponer que la investigación no es la tendencia de las unidades académicas de arte. Un plantel masivo con estudios de maestría se observa a partir de 
la inserción en la academia de los egresados del primer Magíster en Artes Visuales de la UCH, iniciado en 1994.

De las 13 escuelas de arte del sistema nacional universitario, 12 publican la lista completa de sus profesores en la oferta 2012, en las que podemos observar la composición del cuerpo académico según sus grados. De un total de 282 docentes que constituyen el núcleo estable del claustro académico, 17 poseen el grado de doctor (6\%); 63 el de magíster, máster o $\operatorname{DEA}^{5}$ (23,3\%); 162 la licenciatura $(57,4 \%)$ y 33 poseen otros estudios o títulos profesionales $(11,7 \%)$ y 7 declaran no tener grado académico ni título $(2,5 \%)$.

\section{Estudiantes: vacantes, titulados y monto de matrículas}

La lectura de los datos de 2008, publicados en 2009 por SIES ${ }^{6}$, muestra una realidad que no es desconocida ni sorprende a las instituciones: de los 254 titulados en 2008, 183 (72\%) son mujeres y solo 71 son hombres (28\%). Ese mismo año se ofrecieron 603 vacantes para primer año de licenciatura en arte y se matricularon 529 estudiantes $(87,7 \%)$. Del total de matriculados el $68,4 \%$ corresponde a mujeres. En 2010, el número de titulados de licenciatura en arte disminuyó a 216. Con relación a las vacantes, podemos observar una contracción de la oferta, mientras en 2008 se ofrecieron 603 vacantes para primer año de licenciatura en arte, para 2012 se ofrecieron solo 547 cupos, de los cuales se ocupa solamente el $87 \%$.

Si observamos ahora la tasa de titulación, vemos que solo tres programas universitarios reunieron al 56,29\% del total de graduados en 2008. Estos son los programas de la PUC, UFT y UPLA, en el mismo orden. El 43,71\% de los graduados se reparte entre los doce programas restantes. Esta situación sufre una pequeña modificación tres años después: de los 216 graduados en 2010 , el $73 \%$ proviene de tres universidades, a saber, UFT, PUC y UCH.

5 Se excluye como posgrado la Maîtrise, vigente en Francia hasta antes de la Convención de Bolonia, por corresponder a una etapa de pregrado.

6 Bases de datos SIES. Titulados 2008. Consultado en http://www.mineduc.cl/index6. php?id_portal=46\&id_seccion=3222\&id_contenido=13045 
Observemos en la tabla n. 2 el comportamiento de 254 titulados en 2008, que no considera a la UMA ni la UACH (sin datos), y de los 216 del 2010, en el detalle de graduados de licenciatura en arte en el sistema universitario nacional (en orden de mayor a menor):

Tabla n. ${ }^{\circ}$ 2. Titulados / vacantes año 2008

\begin{tabular}{|l|c|c|}
\hline Universidad & $\begin{array}{c}\text { Número de titulados año 2008 } \\
\text { (diversas cohortes) }\end{array}$ & $\begin{array}{c}\text { Vacantes ofrecidas para primer año } \\
\text { (cohorte 2008) }\end{array}$ \\
\hline PUC & 62 & 75 \\
\hline UFT & 46 & 70 \\
\hline UPLA & 35 & 20 \\
\hline ARCIS & 27 & 50 \\
\hline UCH & 22 & 78 \\
\hline UDEC & 17 & 60 \\
\hline UNAB & 12 & 30 \\
\hline UCT & 9 & 30 \\
\hline UMCE & 9 & 20 \\
\hline UNIACC & 6 & 65 \\
\hline UCV & 6 & 30 \\
\hline UDD & 3 & 20 \\
\hline UDP & no hay datos & 35 \\
\hline UMA & no hay datos & 30 \\
\hline UACH & 254 & 30 \\
\hline TOTAL & & 613 \\
\hline
\end{tabular}

Fuente: Bases de datos SIES, Mineduc.

Entre 2008 y 2010, el sistema nacional aumentó levemente la oferta de vacantes universitarias en licenciatura en arte, registrándose un incremento de solo $2,5 \%$ en 24 meses.

Respecto de los montos de matrículas podemos observar que no existe correlación entre el aumento de los valores y el IPC 2010 y 2011, correspondiente al 3,17\% y 4,4\%, respectivamente ${ }^{7}$.

7 Indicadores macroeconómicos Banco Central de Chile. Consultado en http://www.bcentral.cl/ 
228 PERFIL DE LAS LICENCIATURAS EN ARTE DEL SISTEMA UNIVERSITARIO CHILENO - I. Villegas

Tabla n. ${ }^{\circ}$ 3. Valor matrículas años $2010 / 2011 / 2012$

\begin{tabular}{|l|c|c|c|c|c|c|c|}
\hline Universidad & $\begin{array}{c}\text { Valor matrícula } \\
\text { para el año } \\
(1)\end{array}$ & $\begin{array}{c}\text { Valor matrícula } \\
\text { para el ler año } \\
(2)\end{array}$ & $\begin{array}{c}\text { Valor matrícula } \\
1^{\text {er año }}\end{array}$ & \multicolumn{3}{|c|}{ Diferencia respecto de 2010} \\
\cline { 2 - 9 } & 2010 & 2011 & 2012 & \multicolumn{2}{|c|}{2011} & \multicolumn{2}{|c|}{2012} \\
\cline { 2 - 9 } & $\$$ & $\$$ & $\$$ & $\mathrm{M} \$$ & $\%$ & $\mathrm{M} \$$ & $\%$ \\
\hline PUC & 2.870 .000 & 3.040 .000 & 3.200 .000 & +170 & $+5,92$ & +330 & $+11,5$ \\
\hline PUCV & 2.463 .000 & 2.625 .000 & 2.754 .000 & +162 & $+6,58$ & +291 & $+11,8$ \\
\hline UACH & 2.190 .000 & 2.340 .000 & 2.434 .000 & +150 & $+6,85$ & +244 & $+11,1$ \\
\hline UCT & 1.665 .000 & 1.748 .000 & 1.835 .000 & +83 & $+4,98$ & +170 & $+10,21$ \\
\hline UNIACC & 4.932 .000 & 2.690 .000 & 2.800 .000 & -2.242 & $-45,46$ & -2.132 & $-43,2$ \\
\hline ARCIS & 2.479 .000 & 2.349 .000 & 2.870 .000 & -130 & $-5,24$ & +391 & $+15,77$ \\
\hline UCH & 1.878 .000 & 1.878 .000 & 2.093 .700 & 0 & 0 & $+215,7$ & $+11,49$ \\
\hline UDEC & 1.988 .600 & 2.049 .000 & 2.131 .000 & $+60,4$ & $+3,04$ & $+142,4$ & $+7,16$ \\
\hline UPLA & 1.740 .000 & 1.814 .000 & 1.903 .650 & +74 & $+4,25$ & $+163,6$ & $+9,41$ \\
\hline UDD & No hay datos & 4.174 .000 & 4.374 .000 & - & - & - & - \\
\hline UDP & 2.500 .000 & 2.636 .363 & 2.723 .000 & $+136,3$ & $+5,45$ & +223 & $+8,92$ \\
\hline UFT & 3.505 .000 & 3.690 .000 & 3.761 .000 & +185 & $+5,28$ & +256 & $+7,3$ \\
\hline UMA & 3.205 .400 & $\begin{array}{c}\text { No ofrece lic. } \\
\text { en arte }\end{array}$ & $\begin{array}{c}\text { No ofrece lic. } \\
\text { en arte }\end{array}$ & - & - & - & - \\
\hline UMCE & No hay datos & 1.610 .000 & $\begin{array}{c}\text { No ofrece lic. } \\
\text { Arte }\end{array}$ & - & - & - & - \\
\hline UNAB & 2.690 .000 & 2.825 .500 & 2.937 .186 & +135.5 & $+5,04$ & $+247,2$ & $+9,19$ \\
\hline
\end{tabular}

Fuente: (1) Datos oferta académica de pregrado 2010. SIES. Consultado en http://www.mineduc.cl/ index6.php?id_portal=46\&id_seccion=3222\&id_contenido= 13045

(2) Datos consulta telefónica y sitios web en proceso de admisión 2011 y 2012.

Los datos del futuro laboral publicado por SIES en 2009, muestran que al primer año de graduado (cohortes 2005 y 2006) un licenciado en arte puede percibir una renta promedio de $\$ 477.144$, alcanzado el mayor ingreso la suma de $\$ 1.022 .698$ y el más bajo de $\$$ 186.953, es decir, levemente superior al ingreso mínimo mensual de $\$ 172.000$ (julio 2010-julio 2011 ${ }^{8}$ ). El estudio de SIES muestra que al quinto año de egreso un licenciado en arte de las cohortes 2000 y 2001 puede tener un ingreso mensual promedio de $\$ 570.174$, es decir, su sueldo se puede ver incrementado en 19\% (\$93.030) en cinco años. Los estadígrafos muestran en este segmento de egresados una curiosa variación: mientras el promedio de remuneración sube respecto de quienes trabajan por primera vez, habiendo recién egresado, el ingreso mayor esperado para quienes tienen 5 años de experiencia laboral es levemente menor que un recién egresado, llegando a \$ 1.013.285.

8 Ley No 20.449. 
Tabla n. ${ }^{\circ} 4$. Ingresos esperados

\begin{tabular}{|c|c|c|c|c|c|c|c|c|c|}
\hline $\begin{array}{c}\text { Ingresos al ler } \\
\text { año. promedio } \\
\text { mensual } \\
\text { (cohortes 2005 } \\
\text { y 2006) } \\
(\$)\end{array}$ & $\begin{array}{c}10 \% \\
\text { inferior }\end{array}$ & $\begin{array}{c}25 \% \\
\text { inferior }\end{array}$ & $\begin{array}{c}25 \% \\
\text { superior }\end{array}$ & $\begin{array}{c}10 \% \\
\text { superior }\end{array}$ & $\begin{array}{c}\text { Ingresos al 50 } \\
\text { año. promedio } \\
\text { mensual } \\
\text { (cohortes 2000 } \\
\text { y 2001) } \\
(\$)\end{array}$ & $\begin{array}{c}10 \% \\
\text { inferior }\end{array}$ & $\begin{array}{c}25 \% \\
\text { inferior }\end{array}$ & $\begin{array}{c}25 \% \\
\text { superior }\end{array}$ & $\begin{array}{c}10 \% \\
\text { superior }\end{array}$ \\
\hline 477.144 & 186.953 & 231.983 & 545.488 & 1.022 .698 & 570.174 & 231.500 & 310.922 & 738.561 & 1.013 .285 \\
\hline
\end{tabular}

\section{Conclusión}

Metodológicamente, la mayor dificultad para el levantamiento de información radica en la falta de datos contenidos en los sitios web de las instituciones que ofrecen el grado de licenciatura en arte. Las listas de profesores son incompletas; sus grados y las categorías académicas no siempre están a la vista de los interesados. El tipo de jornada de permanencia en los programas no es, definitivamente, una información a la que se puede acceder.

El sistema de educación universitaria ha generado en Chile, para el año 2012, una oferta de 13 programas tendientes a graduar licenciados en arte (la UMCE y la UMA ya no ofrecen el programa). Los programas no poseen rasgos que generen profundas diferencias en la oferta. La estructura de la enseñanza se basa en programas de promedio 4,2 años de duración con mallas curriculares de rasgos comunes: promedio de 45 créditos por semestre; una línea de cursos troncales programados en orden de complejidad o avance, centrados en la producción de obras; talleres de técnicas tradicionales; incorporación de recursos digitales como herramientas de producción de obra; exigencia para graduarse mediante un proyecto de obra y un texto.

Con relación al cuerpo académico, los programas cuentan con un claustro de 25,8 profesores en promedio, en su mayoría con grado académico de licenciado. Solo el 31,2\% de los profesores posee estudios de posgrado, con el menor número de doctores del sistema.

En cuanto al número de vacantes ofrecidas para los programas de licenciatura en arte, la información recolectada permite observar que las universidades que ofrecen la carrera no han variado el tamaño de su oferta en forma significativa en los últimos cinco años. Los datos de 
la muestra obtenida del año 2008 revelan que solo tres universidades concentran más del $50 \%$ de graduados. Cruzado este dato con el número de vacantes ofrecidas históricamente, observamos que no existe una relación entre el número de vacantes ofrecidas y el número de graduados. Es decir, no todos los que ingresan necesariamente se gradúan una vez cumplido el promedio de semestres de duración de la carrera ( 4,2 años). Este rasgo es más cierto en aquellos programas que suelen graduar anualmente más alumnos (de diversas cohortes) que el número de vacantes ofrecidas en cada admisión.

Finalmente, podemos señalar que en la oferta de programas de licenciatura en arte para el año 2011, los valores más bajos de la matrícula correspondieron a la UMCE con $\$ 1.610 .000$, seguida de la UCT con \$1.748.000, mientras que las de más alto valor en sus matrículas son la UDD con $\$ 4.174 .000$ y la UFT con $\$ 3.690 .000$, registrándose una diferencia de $\$ 2.564 .000$ entre el valor de matrícula más alto y el más bajo del sistema. A excepción de la UMCE, que ya no está en la oferta académica, esta tendencia se repite para el año 2012.

Por último, es posible afimar que la oferta de programas de licenciatura en arte que las universidades entregan al mercado nacional, paradójicamente, no es creativa ni variada. Dichos programas poseen rasgos estructurales comunes con la tendencia a reproducir un modelo curricular, lo que se manifiesta en similares procesos de enseñanzaaprendizaje en el aula, sistemas evaluativos y enfoques epistemológicos. Pese a que las universidades manifiestan ideas y posturas diversas, en los programas de licenciatura en arte no encontramos modelos nuevos, disímiles entre sí. No es, por lo tanto, el modelo curricular o el rasgo epistemológico de los contenidos lo que define la selección del programa por los postulantes, quienes probablemente opten según su puntaje PSU, tipo de universidad o valor de la matrícula. Esta uniformidad, como rasgo, ni siquiera se ve alterado en aquellas universidades que poseen posgrados en artes visuales: la PUC y UCH, las cuales podrían ofrecer programas de pregrado con una lógica de continuidad. Los indicadores de éxito que comienzan a aparecer con mediana claridad en el sistema universitario no son considerados en las escuelas de arte a la hora de reformular planes y programas. Sin embargo, asistimos a una modificación del escenario general de la 
enseñanza universitaria, donde tres variables - por lo menos- podrían hacer cambiar la enseñanza del arte en Chile: indicadores de calidad en procesos de acreditación; formación de capital humano avanzado y productividad de los académicos universitarios.

Respecto de la primera variable, podemos señalar que a enero de 2012 solo dos escuelas de arte han obtenido la acreditación: la UNIACC y la UACH, por cuatro años cada una. Mientras que el programa de la PUC espera obtener la acreditación en el transcurso del año 2012. En otras palabras, las escuelas de artes visuales recién se incorporan al manejo de estándares de calidad que podrían eventualmente provocar innovaciones, fruto de la identificación de sus fortalezas y debilidades, y de la elaboración de planes de mejoramiento.

La segunda variable está en permanente tensión con la tercera y cabe preguntarse ¿de qué manera el sistema universitario chileno acoge, en al área de las Artes Visuales, a los posgraduados en esa disciplina? ¿Puede suponerse un futuro mejoramiento de la calidad y nuevas propuestas o enfoques de enseñanza si solamente 29,3\% de los profesores de arte, en el sistema nacional, son posgraduados? El argumento que se esgrime habitualmente es que para ser artista no se necesita tener posgrado. Sin embargo, hablamos aquí de docencia universitaria e investigación, y de creación artística como forma de conocimiento. Podemos suponer que esta misma baja tasa de docentes con posgrado, impide que las escuelas de arte puedan avanzar, por ejemplo, en la ruta en que lo hacen las escuelas de Brasil, México, Estados Unidos o Europa: programas que acogen el desarrollo de obra creativa, docencia e investigación en forma paralela, lo que genera diferenciación teórica, transversalidad en las formas de conocimiento, renovación de modelos curriculares y vinculación entre pregrado y posgrado, es decir, verdadera educación continua actualizada e innovadora. Claramente, hoy en Chile los programas universitarios de artes visuales no están colaborando en forma plena y contundente en la construcción de la sociedad del conocimiento.

Recibido: 17/02/2011

Aceptado: 04/05/2012 